\title{
Percepción de estudiantes de pregrado y evaluación de pares del Consejo Nacional de Acreditación respecto a la calidad de los procesos académicos en una universidad pública*
}

\author{
Claudia María Payán Villamizar** \\ Diana Leal Márquez*** \\ Hoover León Giraldo****
}

Recibido: 3 1-07-2020

Aceptado: 10-08-2020

\begin{abstract}
Citar como: Payán-Villamizar, C. M., Leal-Márquez, D. y León-Giraldo, H. (2021). Percepción de estudiantes de pregrado y evaluación de pares del Consejo Nacional de Acreditación respecto a la calidad de los procesos académicos en una universidad pública. Revista Interamericana de Investigación, Educación y Pedagogía, 14(2), 39-69. https://doi.org/10.15332/25005421.6058
\end{abstract}

\footnotetext{
* Artículo de investigación producto del proyecto de investigación titulado Correlación entre la percepción de los estudiantes de pregrado y la evaluación de pares del Consejo Nacional de Acreditación (CNA) respecto a la calidad de los procesos académicos, financiado por la Universidad del Valle.

** Terapeuta ocupacional, magíster en Administración de Salud y especialista en Docencia Universitaria. Docente titular de la Facultad de Salud de la Universidad del Valle. Directora de Autoevaluación y Calidad Académica de la Universidad del Valle. Miembro del grupo de investigación Sinergia de la línea de investigación Interacciones educativas en torno a la discapacidad.

Correo electrónico: claudia.payan@correounivalle.edu.co

ORCID: https://orcid.org/0000-0002-4603-1403

Google Scholar: https://scholar.google.es/citations?view_op=list_works\&hl=es\&user=gOSW49YAAAAJ

Registro CvLac: https://scienti.minciencias.gov.co/cvlac/visualizador/generarCurriculoCv.do?cod_rh=0000530646

*** Ingeniera industrial y especialista y magíster en Administración. Profesional de la Dirección de Autoevaluación y Calidad Académica de la Universidad del Valle. Miembro del grupo de investigación Sinergia de la línea de investigación Interacciones educativas en torno a la discapacidad.

Correo electrónico: diana.leal@correounivalle.edu.co

ORCID: https://orcid.org/0000-0001-6164-7973

Google Scholar: https://scholar.google.es/citations?user=S2nfWOQAAAAJ\&hl=es

Registro CvLac: http://scienti.colciencias.gov.co:8081/cvlac/visualizador/generarCurriculoCv.do?cod_rh=00001 13748

***** Estadístico. Profesional de la Dirección de Autoevaluación y Calidad Académica de la Universidad del Valle. Miembro del grupo de investigación Sinergia de la línea de investigación Interacciones educativas en torno a la discapacidad.

Correo electrónico: hoover.leon@correounivalle.edu.co

ORCID: https://orcid.org/0000-0002-8854-105X

Google Scholar: https://scholar.google.com/citations?user=15aaG6oAAAAJ\&hl=en

Registro CvLac: http://scienti.colciencias.gov.co:8081/cvlac/visualizador/generarCurriculoCv.do?cod_rh=0000541 184
} 


\section{Resumen}

Objetivo: relacionar la percepción de estudiantes de pregrado y la evaluación realizada por pares académicos del Consejo Nacional de Acreditación en relación con el factor procesos académicos, como resultado de la autoevaluación con fines de acreditación en alta calidad. Metodología: el estudio fue observacional, descriptivo, transversal y correlacional. Se analizó la autoevaluación de 3408 estudiantes de 25 programas académicos entre 2014 y 2018, y se comparó con el informe de los pares académicos del Consejo Nacional de Acreditación con respecto al grado de cumplimiento de las características de los programas académicos. Se evaluaron las características que tuvieron mayor variabilidad: apoyo docente, interdisciplinariedad, evaluación y autorregulación, y recursos informáticos. La correlación se analizó utilizando el índice kappa y se consideró un valor de referencia menor de 0.05. Resultados: se encontró una concordancia débil entre las dos evaluaciones con respecto a la calidad de los procesos académicos ( $K=0.242$ ). Los pares académicos otorgaron una mejor calificación en las cinco categorías evaluadas. Conclusión: existe diferencia entre la percepción de los estudiantes de pregrado y la evaluación de los pares académicos con respecto a las características del factor procesos académicos, que fueron mejor evaluadas por los pares que por los estudiantes.

Palabras clave: autoevaluación, acreditación, calidad de la educación, evaluación de la educación, educación superior, percepción. 


\title{
Perception of Undergraduate Students and Peer Evaluation of the National Accreditation Council Regarding the Quality of Academic Processes in a Public University
}

\begin{abstract}
Objective: to relate the perception of undergraduate students and the evaluation carried out by academic peers of the National Accreditation Council in light of the academic processes factor, as a result of the self-evaluation processes for high-quality accreditation purposes. Methodology: the study was observational, descriptive, cross-sectional, and correlational. The self-evaluation of 3.408 students from 25 academic programs between 2014 and 2018 was analyzed and compared with the report of the academic peers of the National Accreditation Council, regarding the degree of compliance with the characteristics of academic programs. The characteristics that had greater variability were evaluated: teaching support, interdisciplinarity, evaluation and self-regulation, and computer resources. The correlation was analyzed using the kappa index and a reference value of less than 0.05 was considered. Results: a weak concordance was found between the two evaluations regarding the quality of the academic processes $(K=0.242)$. Academic peers gave a better grade in the five categories evaluated. Conclusion: there is a difference between the perception of undergraduate students and the evaluation of academic peers regarding the characteristics of the academic processes factor, which was better evaluated by peers than by students.
\end{abstract}

Keywords: self-evaluation, accreditation, quality of education, educational evaluation, higher education, perception. 


\section{Percepção de estudantes de graduação e avaliação por pares do Conselho Nacional de Acreditação quanto a qualidade dos processos acadêmicos em uma universidade pública}

\section{Resumo}

Objetivo: relacionar a percepção dos alunos de graduação e a avaliação realizada pelos pares acadêmicos do Conselho Nacional de Acreditação em relação ao fator "Processos Acadêmicos" em decorrência dos processos de autoavaliação para fins de acreditação de alta qualidade. Metodologia: o estudo foi observacional, descritivo, transversal e correlacional. Foi analisada a autoavaliação de 3.408 alunos de 25 programas acadêmicos entre 2014 e 2018 e comparada com o relatório dos pares acadêmicos do Conselho Nacional de Acreditação, quanto ao grau de cumprimento das características dos programas acadêmicos. Foram avaliadas as características que apresentaram maior variabilidade: suporte didático, interdisciplinaridade, avaliação e autorregulação, e recursos de informática. A correlação foi analisada pelo índice kappa e considerado um valor de referência menor que 0,05. Resultados: foi encontrada fraca concordância entre as duas avaliações quanto à qualidade dos processos acadêmicos $(K=0,242)$. Os pares acadêmicos deram melhores notas nas cinco categorias avaliadas. Conclusão: há diferença entre a percepção dos alunos de graduação e a avaliação dos pares acadêmicos quanto às características do fator "processos acadêmicos", sendo melhor avaliada pelos pares do que pelos alunos.

Palavras-chave: autoavaliação, acreditação, qualidade da educação, avaliação educacional, educação superior, percepção. 


\section{Introducción}

El concepto de calidad se define como la "propiedad o conjunto de propiedades inherentes a algo, que permiten juzgar su valor", "buena calidad, superioridad o excelencia", "adecuación de un producto o servicio a las características especificadas" (Real Academia Española, 2019, definiciones 1, 2 y 3). Lo anterior deja entrever que la calidad es un término relativo multidimensional, que supone acepciones diferentes tanto en el tiempo como en función de quien lo utilice, pues implica el necesario juicio de valor individual y colectivo (Colmenares Delgado y Saavedra Torres, 2007).

En el proceso evolutivo del concepto de calidad se distinguen diversas etapas y enfoques, producto de las teorías de expertos como Feigenbaum, Jurán, Cosby, Shewhart y Deming, entre las que se resalta el enfoque de aseguramiento de la calidad, cuya su finalidad es comprobar que se realizan las actividades satisfactoriamente, de modo que el producto resultante sea adecuado (Saumeth et ál., 2012). Este concepto se nutre a través de otros enfoques como el de calidad total, en el cual en cada fase del proceso se deben identificar las necesidades del cliente. Este concepto es Ilevado al contexto de la educación para efectos del seguimiento en la percepción de los estudiantes en relación con los servicios educativos que le son ofertados.

En la actualidad la calidad no está únicamente enfocada a un producto o servicio, sino que también involucra a las personas que trabajan en las organizaciones y requieren de las competencias necesarias para afrontar grandes desafíos. Aquí se incluyen a las instituciones de educación superior, sobre las que recae la responsabilidad de generar y difundir el conocimiento en la sociedad (Aguerrondo, 1993).

El concepto de calidad no es absoluto, sino relativo. Este lleva implícito el de apreciación o evaluación, lo cual es necesario hacer en función de ciertas normas o estándares preestablecidos, que permitan juzgar su mayor o menor adecuación a los patrones 
o modelos de referencia. Calidad, evaluación y acreditación son conceptos interrelacionados (Tünnermann 2006). Según el Acuerdo 02 de 2020 del Consejo Nacional de Educación Superior (CESU):

La acreditación en alta calidad promueve el fortalecimiento de una cultura de la alta calidad de los programas académicos y de las instituciones. La evaluación con fines de acreditación en alta calidad se realiza por la institución en sí misma y sus programas académicos, los pares académicos y el Consejo Nacional de Acreditación.

La autoevaluación con fines de acreditación en alta calidad de programas académicos es el proceso mediante el cual se evalúan sus fortalezas y oportunidades de mejora, con el objetivo de identificar aspectos que permitirán avanzar en su calidad. Esto a través del análisis interno de las actividades, la participación de la comunidad universitaria y la evaluación externa realizada por los pares académicos, quienes recomiendan o no la acreditación ante el Ministerio de Educación Nacional. Se autoevalúan aspectos como el proyecto educativo, las actividades académicas de los estudiantes, la calidad de los docentes, los procesos de investigación, la oferta de bienestar universitario, la infraestructura y los mecanismos de seguimiento a egresados.

La finalidad del proceso de evaluación es que los resultados obtenidos sirvan de apoyo para la toma de decisiones en la determinación de políticas, estrategias y acciones para el mejoramiento académico de las instituciones (Ramos, 2007). La mejora es el objetivo de la acreditación que se menciona con mayor frecuencia, ya que se refiere exactamente a lo que uno esperaría del aseguramiento de la calidad: ayudar a las instituciones a adquirir las condiciones necesarias, mejorar los procesos y aumentar la calidad de los resultados (Cuevas et ál., 2019).

En nuestro país, la Política de Aseguramiento de la Calidad plantea cambios que buscan - desde la transformación curricular y de la evaluación - renovar los programas, diseñar procesos educativos y curriculares interactivos, imponer nuevos modelos de gestión 
educativa, construir nuevos paradigmas educativos, entre otros aspectos (Gallego, 2012).

Por ello, los procesos académicos relacionados con la formación de estudiantes son misionales y fundamentales del quehacer de las instituciones de educación superior. Al ser los estudiantes usuarios de los servicios académicos, administrativos, culturales, sociales y de bienestar ofrecidos por las instituciones, estos son permanentemente consultados como uno de los principales actores del proceso. Por lo tanto, su formación está directamente relacionada con los servicios educativos que reciben y la forma en que los apropian a su desarrollo.

Generalmente, la percepción sobre la calidad de un programa académico se puede reconocer en la calidad académica de la formación que reciben los estudiantes y la manera como estos son formados en competencias, actitudes, conocimientos, capacidades, habilidades y destrezas para su desempeño profesional. Esto se puede evidenciar en el factor 4 de los lineamientos del Consejo Nacional de Acreditación (CNA) relacionado con los procesos académicos, el cual menciona que "un programa de alta calidad se reconoce por la capacidad que tiene de ofrecer una formación integral, flexible, actualizada e interdisciplinar, acorde con las tendencias contemporáneas del área disciplinar o profesional que le ocupa" (Consejo Nacional de Acreditación [CNA], 2013, p. 29).

La calidad en el sector educativo se asocia con el cumplimiento de estándares en relación con el nivel de educación recibido por los estudiantes y con la formación académica de los docentes, los procesos investigativos, las relaciones de cooperación y el impacto de sus egresados en el medio. De esta manera se asocia la calidad al aseguramiento de esta, de tal forma que sea sostenida en el tiempo de acuerdo con las condiciones, contexto y naturaleza de las instituciones de educación superior. Esto con el propósito de lograr una cultura de autorregulación que oriente al mejoramiento continuo de las instituciones y programas, a través de un trabajo articulado con la comunidad universitaria (Estrada, 1999; Ferrer, 2006; Giraldo, Abad y Díaz, 2007; Calvo, 2018; Bonfante, 2019). 
El concepto de calidad aplicado al bien público de la educación superior hace referencia a la síntesis de características que permiten reconocer un programa académico específico o una institución de determinado tipo, y hacer un juicio sobre la distancia relativa entre el modo como en esa institución o en ese programa académico se presta dicho servicio y el óptimo que corresponde a su naturaleza (CNA, 2013, p. 12).

En este punto se retoma la idea de que la calidad siempre estará articulada con el cumplimiento de una serie de expectativas de la comunidad, la cual espera satisfacer cierto grado de necesidades relacionadas, en este caso con la educación: contar con docentes de alta calidad; tener un currículo con asignaturas y cursos que permitan a los egresados desarrollar habilidades y capacidades para destacarse en un entorno globalizado como el actual; tener una formación integral que le permita desempeñarse en cualquier área, con las competencias adecuadas para ello; entre otros aspectos.

El CNA - como organismo con funciones de coordinación, planificación, recomendación y asesoría en el tema de acreditación de programas y de instituciones de Educación Superior en Colombia y que depende del CESU - ha definido, en conjunto con organizaciones y entidades nacionales, una serie de lineamientos para orientar a las instituciones y sus programas académicos en la evaluación de sus condiciones académicas, tanto a nivel institucional como para todos los niveles de formación pregrado, posgrado (maestrías y doctorados) y especialidades médicas; todos ellos con características específicas y de acuerdo con sus particularidades formativas.

Estas condiciones académicas están agrupadas en factores (pilares para la valoración de los programas académicos vistos desde una percepción sistémica), características (que expresan referentes universales y particulares de la calidad) y aspectos a evaluar (que corresponden a aspectos cualitativos o cuantitativos para hacer observable y valorable el grado de calidad alcanzado) (CNA, 2013, pp. 17-18), que brindan la orientación para alcanzar la acreditación de alta calidad de acuerdo con el cumplimiento de sus condiciones. 
En el marco de la autonomía universitaria, las instituciones deben: definir los conceptos que se asocian con las condiciones académicas según sus propósitos, misión, visión y principios institucionales; ser debidamente divulgados y conocidos por la comunidad educativa; y ser coherentes con el proyecto educativo institucional. En este punto lo más importante es evidenciar su correspondencia con los proyectos educativos de los programas, los cuales orientan los fines de la formación académica, que incluyen - de una manera u otra - los aspectos antes mencionados, para garantizar la prestación de servicios educativos según los requerimientos y necesidades de la sociedad, y el cumplimiento de los estándares nacionales.

Por lo tanto, la autoevaluación con fines de acreditación en alta calidad tiene como propósito evaluar los servicios -en este caso de las condiciones de calidad de los procesos académicosy determinar acciones para el mejoramiento continuo. En el marco de la autonomía universitaria, este proceso se realiza a través de diferentes medios y con la participación de diferentes actores: 1) al interior de la institución, la comunidad académica (directivos, docentes, estudiantes, graduados, empleadores, personal administrativo), quienes realizan una valoración frente a la percepción de cómo se encuentran las condiciones de calidad, y el comité de autoevaluación, que analiza su comportamiento en el tiempo; y 2) al exterior de la institución, los pares académicos, quienes examinan la forma como un programa cumple con las exigencias de calidad establecidas por la comunidad académica de la profesión o disciplina.

El análisis de la percepción de la calidad del servicio por parte de los estudiantes es de gran interés para las instituciones de educación superior, pues compiten por ofrecer el mejor servicio de educación, pero desconocen lo que piensan los usuarios al respecto. La opinión y percepción del estudiante acerca del servicio que está recibiendo es importante, porque él es el cliente que juzga el servicio. La evaluación de la calidad del servicio en las universidades es fundamental para ejercer un control en los procedimientos que se llevan a cabo y para que se puedan mejorar o perfeccionar 
funciones que se realizan mal; además, debe ser constante, llevar un seguimiento y ser automática. Entendida de este modo, la calidad requiere un juicio valorativo que viene dado por la evaluación. Es importante hacer la distinción entre calidad y evaluación, pues mientras la calidad de la educación implica un proceso de mejora continuo sobre sus elementos, también requiere necesariamente de la evaluación (Reyes Sánchez y Reyes Pazos, 2012).

Por su parte, la función del par académico se basa en la verificación de la coherencia entre lo que el informe de autoevaluación expresa y la evidencia durante la visita a la institución, más específicamente al programa. En esta verificación emite juicios de calidad, basados no solamente en el informe de autoevaluación, sino en aquellos aspectos encontrados durante la visita de evaluación externa; y recomienda a las entidades acreditadoras el reconocimiento de la calidad o no de las instituciones y programas. Es allí donde se conjugan la autoevaluación, la evaluación externa, la evaluación integral y la acreditación, todas como modalidades de evaluación (Selley y Ramírez, 2018).

Las fases que debe cumplir un par académico para la elaboración del informe de evaluación externa son:

- La ponderación: es el análisis del deber ser de la profesión o la disciplina en consonancia con la misión institucional y en el marco de las tendencias nacionales e internacionales, el cual es realizado por la comunidad académica (en el caso de la Universidad del Valle, la ponderación es realizada por el comité del programa o el comité de autoevaluación). Esto posibilita observar el desempeño en un escenario determinado, a través de los factores y características establecidos por el CNA.

- La emisión de juicios: es la dimensión del ser en el programa, y es cuando el par académico le otorga un valor a cada una de las características con base en el análisis de la información cuantitativa, cualitativa y estadística. 
- Los planes de mejoramiento: se construyen a partir de la emisión de juicios de las características y factores, y sirven para continuar con la ruta de la excelencia (CNA, 2016).

Es pertinente entonces - si se requiere hablar de mejoramiento continuo en procesos de calidad educativa - analizar la coherencia, la consistencia y los aspectos involucrados en la valoración de los procesos académicos por parte de los estudiantes, así como el grado de conocimiento que tienen acerca de conceptos como interdisciplinariedad, integralidad, flexibilidad, evaluación y autorregulación. Este análisis se relaciona con la evaluación que los pares otorgan a los procesos académicos, quienes - a través del análisis de fortalezas y oportunidades de un programa académico- contribuyen al mejoramiento de su calidad. Aunque no se encuentran referentes conceptuales sobre los procesos académicos, se deduce, por lo establecido en los lineamientos del CNA, que estos se refieren al currículo o al proyecto educativo de un programa académico. Por lo tanto, incluyen la misión y los objetivos, los contenidos organizados en planes de estudio, los aspectos metodológicos para la enseñanza y el aprendizaje, los materiales didácticos y la evaluación (Ruiz et ál., 2015).

Según Ramírez (2011), apreciar (dar valor a la calidad) es una labor subjetiva; por ello, la investigación cualitativa se afirma desde los supuestos e intereses desde los que se sustenta. Se constituye en un modo particular de acercarse a la realidad, en este caso la educativa, que busca interpretarla para comprenderla y así orientar la acción de los actores de las comunidades educativas de manera que sus acciones sean razonadas y responsables.

El objetivo de la presente investigación fue relacionar la percepción de estudiantes de pregrado y la evaluación realizada por pares académicos del Consejo Nacional de Acreditación con respecto al factor procesos académicos como resultado de los procesos de autoevaluación con fines de acreditación en alta calidad. 


\section{Metodología}

Se realizó un estudio observacional descriptivo de corte transversal y correlacional. Se analizaron los reportes de autoevaluación con fines de acreditación diligenciados por 3408 estudiantes de 25 programas académicos de pregrado, en el periodo 2014-2018, y el informe de evaluación externa emitido por los pares académicos del CNA después de la visita a cada uno de dichos programas.

Se analizaron las siguientes variables: integralidad del currículo, flexibilidad del currículo, interdisciplinariedad, estrategias de enseñanza y aprendizaje, sistema de evaluación de estudiantes, evaluación y autorregulación del programa, recursos bibliográficos, recursos informáticos y de comunicación, y recursos de apoyo docente. Estas variables contienen la calificación generada tanto por los estudiantes, como por los pares académicos; sin embargo, en la metodología de evaluación se considera el uso de aspectos a evaluar tanto cuantitativos como cualitativos (con el fin de demostrar el nivel de calidad que se está probando). La calificación cuantitativa está en un rango de 1 a 5 , la cual se traduce en una valoración cualitativa de la siguiente manera: plenamente (4.5-5), alto grado (3.8-4.4), aceptable (3-3.7) e insatisfactorio (1-2.9). Ambas valoraciones se compararon con la realizada por los pares académicos.

Las variables continuas se expresaron en promedios y desviación estándar; y para las variables cualitativas se estimaron proporciones. Se estimó el coeficiente de variación (CV, relación entre la desviación estándar y el promedio de las calificaciones) para analizar qué tanto se distancian las calificaciones de cada característica con respecto a la calificación promedio total. Por su parte, el análisis de la relación entre los resultados de la percepción de los estudiantes y los resultados de la evaluación externa del CNA se realizó mediante el índice de kappa, para establecer concordancias y acuerdos. 
Inicialmente se presentan los resultados relacionados con la percepción de los estudiantes de pregrado sobre las características del factor procesos académicos, luego se describen los resultados de la evaluación externa del CNA con respecto al mismo factor. Con base en lo anterior, se presenta el análisis de concordancia entre la relación de los resultados de la percepción de los estudiantes y los resultados de la evaluación externa del CNA. Finalmente, se señala la conceptualización que tienen los estudiantes de pregrado sobre los elementos que componen los procesos académicos.

Todos los análisis cuantitativos se realizaron con el paquete estadístico Stata $11.2{ }^{\circledR}$ y Microsoft Office Excel 2013 para Windows.

\section{Resultados}

\section{Percepción de los estudiantes}

La calificación general del factor procesos académicos obtuvo una valoración de alto grado, cuya calificación promedio fue de 3.8, un valor mínimo de 2.9 (insatisfactorio) y un máximo de 4.6 (alto grado). En 10 de los 25 programas (37\%) la valoración fue insatisfactoria. La calificación de las características evaluadas por los estudiantes se presenta en la tabla 1 , cuyo juicio sobre su calidad se expresa en escalas no numéricas definidas como: plenamente, alto grado, aceptable e insatisfactorio.

Tabla 1. Calificación y escala de valoración no numérica de la autoevaluación de los estudiantes frente a las características de los procesos académicos (orden descendente por promedio).

\begin{tabular}{|c|c|c|c|c|c|c|}
\hline \multirow[b]{2}{*}{ Característica } & \multicolumn{5}{|c|}{ Calificación } & \multirow{2}{*}{$\begin{array}{l}\text { Escala no } \\
\text { numérica }\end{array}$} \\
\hline & Mínimo & Máximo & $\begin{array}{l}\text { Desviación } \\
\text { estándar }\end{array}$ & Promedio & $\mathbf{C V}^{*}$ & \\
\hline Recursos informáticos & 3.7 & 4.7 & 0.277 & 4.17 & $6.6 \%$ & Alto grado \\
\hline Evaluación & 2.8 & 4.8 & 0.45 & 4.10 & $11.0 \%$ & Alto grado \\
\hline Metodologías & 2.9 & 4.8 & 0.548 & 4.04 & $13.6 \%$ & Alto grado \\
\hline Integralidad & 2.8 & 4.8 & 0.626 & 3.98 & $15.7 \%$ & Alto grado \\
\hline Recursos bibliográficos & 3.1 & 4.7 & 0.465 & 3.94 & $11.8 \%$ & Alto grado \\
\hline Interdisciplinariedad & 2.5 & 4.8 & 0.66 & 3.65 & $18.1 \%$ & Aceptable \\
\hline
\end{tabular}




\begin{tabular}{|l|c|c|c|c|c|c|}
\hline \multirow{2}{*}{\multicolumn{1}{|c|}{ Característica }} & \multicolumn{5}{c|}{ Calificación } & Escala no \\
\cline { 2 - 7 } & Mínimo & Máximo & $\begin{array}{c}\text { Desviación } \\
\text { estándar }\end{array}$ & Promedio & CV*a \\
\hline Flexibilidad & 2.5 & 4.5 & 0.519 & 3.59 & $14.5 \%$ & Aceptable \\
\hline Apoyo docente & 2 & 4.5 & 0.685 & 3.59 & $19.1 \%$ & Aceptable \\
\hline $\begin{array}{l}\text { Evaluación y } \\
\text { autorregulación }\end{array}$ & 2.5 & 4.5 & 0.624 & 3.48 & $17.9 \%$ & Aceptable \\
\hline Factor (total) & 2.8 & 4.7 & & 3.8 & & Alto grado \\
\hline
\end{tabular}

${ }^{*} \mathrm{CV}$ : coeficiente de variación.

Fuente: elaboración propia.

De acuerdo con el coeficiente de variación, la mayor variabilidad se presentó en el apoyo docente, interdisciplinariedad y evaluación y autorregulación. Mientras que la característica de recursos informáticos presentó la menor variación (figura 1).

Figura 1. Variación de las calificaciones de los estudiantes con respecto a las características de los procesos académicos.

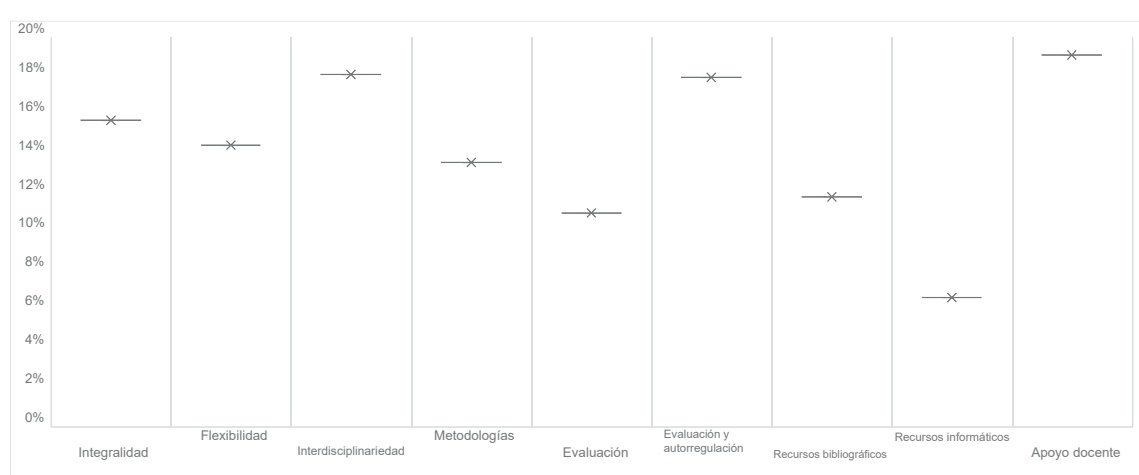

Fuente: elaboración propia.

Al analizar por separado estas tres características, se observó que el apoyo docente tuvo una calificación aceptable por parte de los estudiantes, correspondiente a 3.59. Los resultados de las encuestas para esta característica en términos de disponibilidad, calidad y uso de los recursos audiovisuales por parte de los docentes, mostró que en promedio el porcentaje de los estudiantes dieron calificaciones de excelente y buena; con respecto a la disponibilidad fue del $54 \%$, en la calidad de $53 \%$ y en el uso de recursos audiovisuales de $66.4 \%$. En otros programas una mayor 
proporción de estudiantes calificaron $83 \%$ para disponibilidad, $79.4 \%$ para calidad y $87.3 \%$ para al uso de recursos audiovisuales. En cuanto a los resultados desfavorables se evidenció que en promedio el porcentaje de estudiantes que dieron calificaciones de regular y malo a la disponibilidad fue del $41.8 \%$, en la calidad de $43.8 \%$ y en el uso de recursos audiovisuales fue del $29 \%$ (figuras 2,3 y 4 ).

Figura 2. Proporción de estudiantes que calificaron la disponibilidad de los recursos audiovisuales docentes.

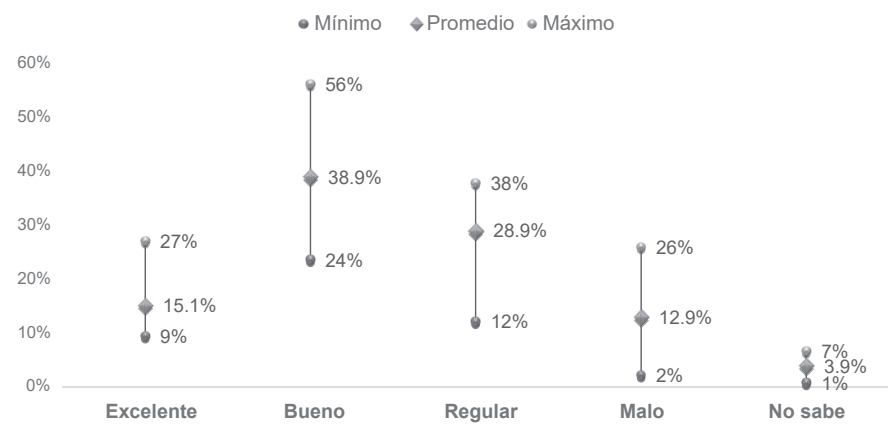

Fuente: elaboración propia.

Figura 3. Proporción de estudiantes que calificaron la calidad de los recursos audiovisuales docentes.

- Mínimo $\diamond$ Promedio • Máximo

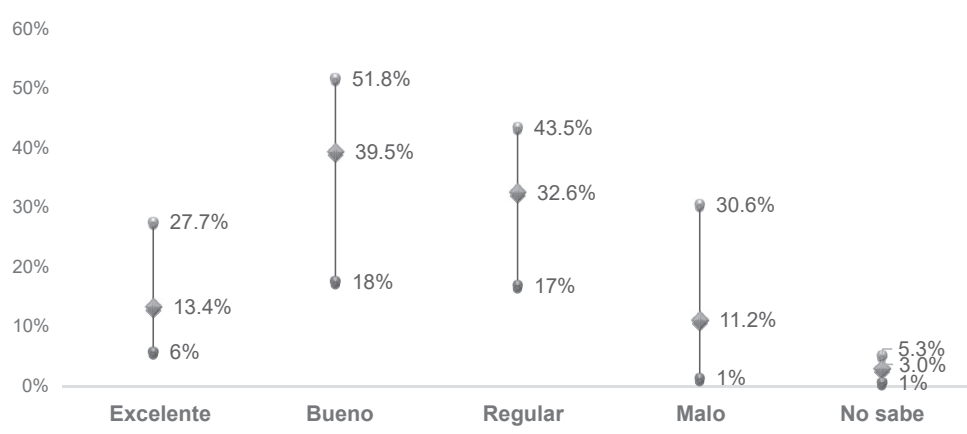

Fuente: elaboración propia. 
Figura 4. Proporción de estudiantes que calificaron el uso de los recursos audiovisuales docentes.

- Mínimo $\diamond$ Promedio • Máximo

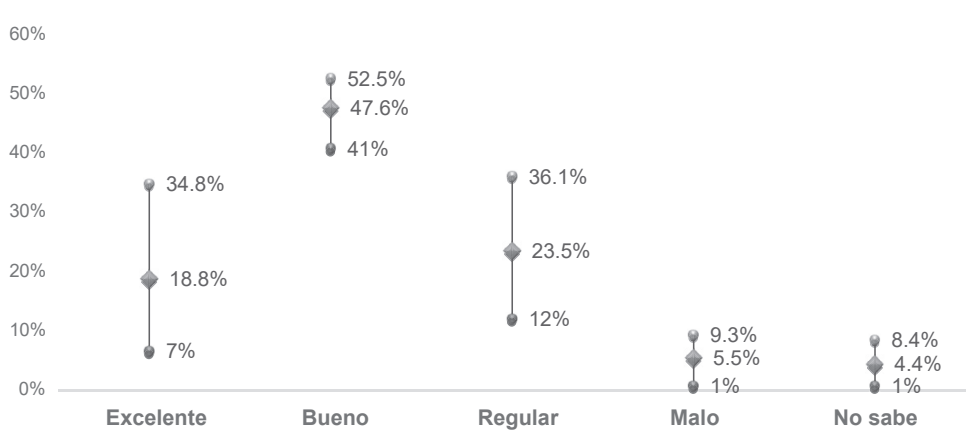

Fuente: elaboración propia.

La interdisciplinariedad -que se evalúa con respecto a las actividades curriculares y extracurriculares que se realizan en el programa o la universidad, donde se califica su pertinencia y eficacia al presentar resultados de favorabilidad o desfavorabilidad por parte de los estudiantes - tuvo una calificación aceptable (3.65). En promedio, el $59.4 \%$ de los estudiantes calificaron la pertinencia entre excelente y buena; en algunos programas una mayor proporción de estudiantes lo calificaron en $85.8 \%$. En relación con la eficacia, los resultados fueron similares: en promedio, el $52.4 \%$ de los estudiantes dieron una calificación entre excelente y buena, mientras que en algunos programas una mayor proporción de estudiantes dieron una calificación de $83.7 \%$. En cuanto a los resultados desfavorables se evidenció que, en promedio, el porcentaje de estudiantes que dieron calificaciones de regular y malo a la pertinencia de las actividades curriculares y extracurriculares fue del $26.4 \%$, y en relación con la eficacia fue del $33.7 \%$ (figuras 5 y 6). 
Figura 5. Proporción de estudiantes que calificaron la pertinencia de las actividades de tipo interdisciplinario (curriculares y extracurriculares).

- Mínimo $\diamond$ Promedio • Máximo

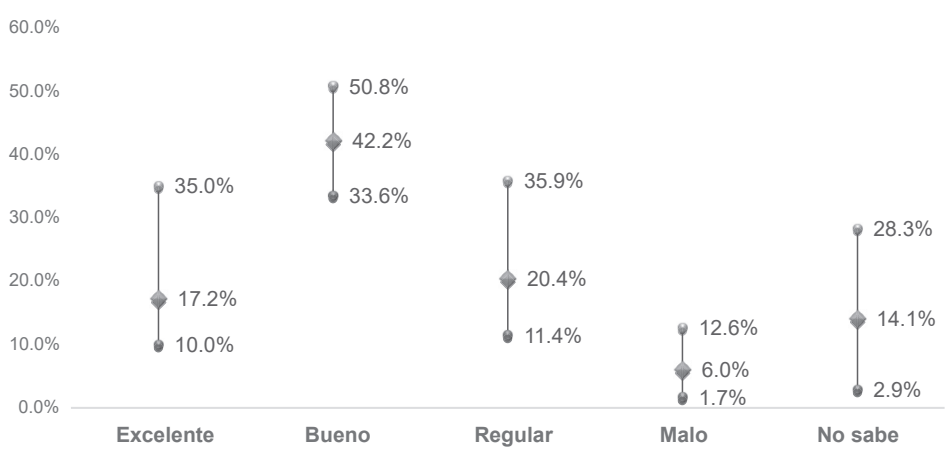

Fuente: elaboración propia.

Figura 6. Proporción de estudiantes que calificaron la eficacia sobre las actividades de tipo interdisciplinario (curriculares y extracurriculares).

- Mínimo $\diamond$ Promedio @ Máximo

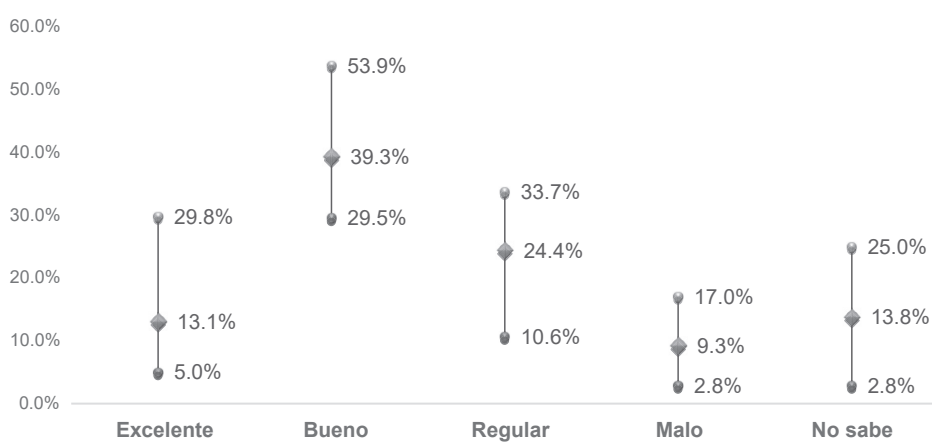

Fuente: elaboración propia.

Finalmente, se evaluó el nivel de acuerdo en relación con la contribución de los sistemas de evaluación y autorregulación del programa en el mejoramiento de su calidad. La calificación general dada por los estudiantes igualmente fue aceptable (3.48). Los resultados favorables mostraron que, en promedio, el $51.5 \%$ de los estudiantes están totalmente y en gran medida de acuerdo 
con la contribución de estos sistemas en el mejoramiento de la calidad del programa académico. En otros programas este mismo nivel se observó en una proporción mayor de estudiantes, correspondiente al $82 \%$. En relación con los resultados desfavorables, se presenta que, en promedio, el $37.9 \%$ de los estudiantes estuvieron parcialmente de acuerdo o en desacuerdo con dicha contribución (figura 7).

Figura 7. Proporción de estudiantes que calificaron el nivel de acuerdo en relación con los sistemas de evaluación y autorregulación del programa.

• Mínimo $\diamond$ Promedio • Máximo

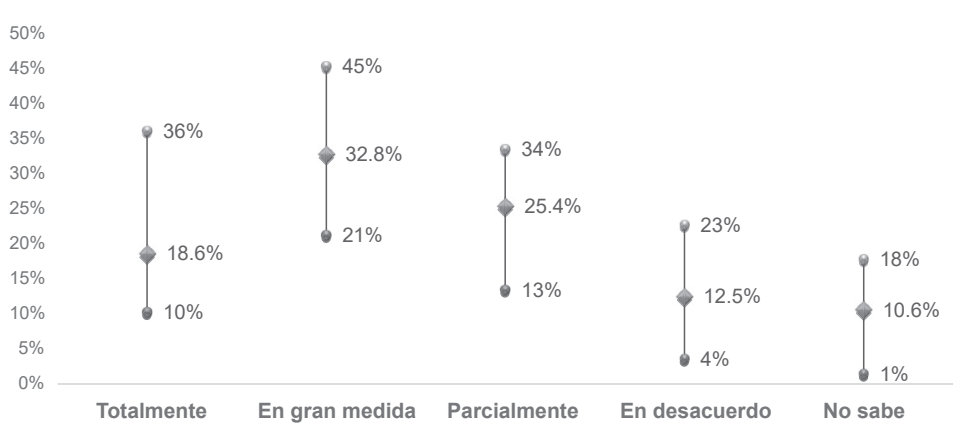

Fuente: elaboración propia.

La característica recursos informáticos fue la que presentó menor variabilidad en comparación con las demás. Esto se explica porque obtuvo la mayor calificación, con un promedio de 4.17 (alto grado) y una calificación mínima de 3.7 (superior que las otras características) y una calificación máxima de 4.7 (similar a las demás).

\section{Resultados de la evaluación externa del CNA}

La calificación de los pares académicos para el factor procesos académicos alcanzó una valoración de alto grado, con un promedio de 4.1, un valor mínimo de 3.7 (aceptable) y un máximo de 4.5 (plenamente). En el $67 \%$ de los programas (18) la valoración fue de alto grado.

La calificación de la evaluación externa contempló las once características que componen el factor de procesos académicos; 
sin embargo, los pares emitieron una calificación cuantitativa por característica en solo seis programas y otorgaron una valoración (plenamente, alto grado y aceptable) del factor en cada programa. La calificación de la evaluación externa en los seis programas se presenta en la tabla 2, cuyo juicio sobre su calidad se expresa en escalas no numéricas definidas como plenamente, alto grado, aceptable e insatisfactorio.

Tabla 2. Calificación y escala de valoración no numérica de la evaluación externa frente a las características de los procesos académicos.

\begin{tabular}{|c|c|c|c|c|c|c|}
\hline \multirow[b]{2}{*}{ Característica } & \multicolumn{5}{|c|}{ Calificación } & \multirow{2}{*}{$\begin{array}{l}\text { Escala no } \\
\text { numérica }\end{array}$} \\
\hline & Mínimo & Máximo & $\begin{array}{l}\text { Desviación } \\
\text { estándar }\end{array}$ & Promedio & $\mathrm{CV}^{*}$ & \\
\hline Integralidad & 3.8 & 4.7 & 4.27 & 0.38 & $8.8 \%$ & Alto grado \\
\hline Flexibilidad & 3.5 & 4.6 & 4.08 & 0.43 & $10.6 \%$ & Alto grado \\
\hline Interdisciplinariedad & 2.3 & 4.7 & 3.9 & 0.84 & $21.6 \%$ & Alto grado \\
\hline Metodologías & 3.6 & 4.8 & 4.32 & 0.42 & $9.8 \%$ & Alto grado \\
\hline Evaluación & 3.9 & 4.7 & 4.22 & 0.32 & $7.6 \%$ & Alto grado \\
\hline Trabajos & 4.0 & 4.8 & 4.42 & 0.29 & $6.5 \%$ & Alto grado \\
\hline $\begin{array}{l}\text { Evaluación y } \\
\text { autorregulación }\end{array}$ & 3.4 & 4.8 & 3.92 & 0.62 & $15.8 \%$ & Alto grado \\
\hline Extensión & 3.6 & 4.8 & 4.17 & 0.42 & $10.1 \%$ & Alto grado \\
\hline Recursos bibliográficos & 4.1 & 4.6 & 4.44 & 0.19 & $4.4 \%$ & Alto grado \\
\hline Recursos informáticos & 3.7 & 4.6 & 4.14 & 0.36 & $8.6 \%$ & Alto grado \\
\hline Apoyo docente & 3.0 & 4.3 & 3.82 & 0.52 & $13.5 \%$ & Alto grado \\
\hline Factor & 3.7 & 4.5 & 4.10 & & & Alto grado \\
\hline
\end{tabular}

${ }^{*} \mathrm{CV}$ : coeficiente de variación.

Fuente: elaboración propia.

Con base en las calificaciones promedio de cada una de las características anteriores, se calculó el coeficiente de variación para analizar qué tanto estas se distancian con respecto a la calificación promedio total. Es así como la mayor variación se presentó en interdisciplinariedad y evaluación y autorregulación; y la menor variación se presentó en recursos bibliográficos (figura 8). 
Figura 8. Variación de las calificaciones de la evaluación externa respecto a las características de los procesos académicos.

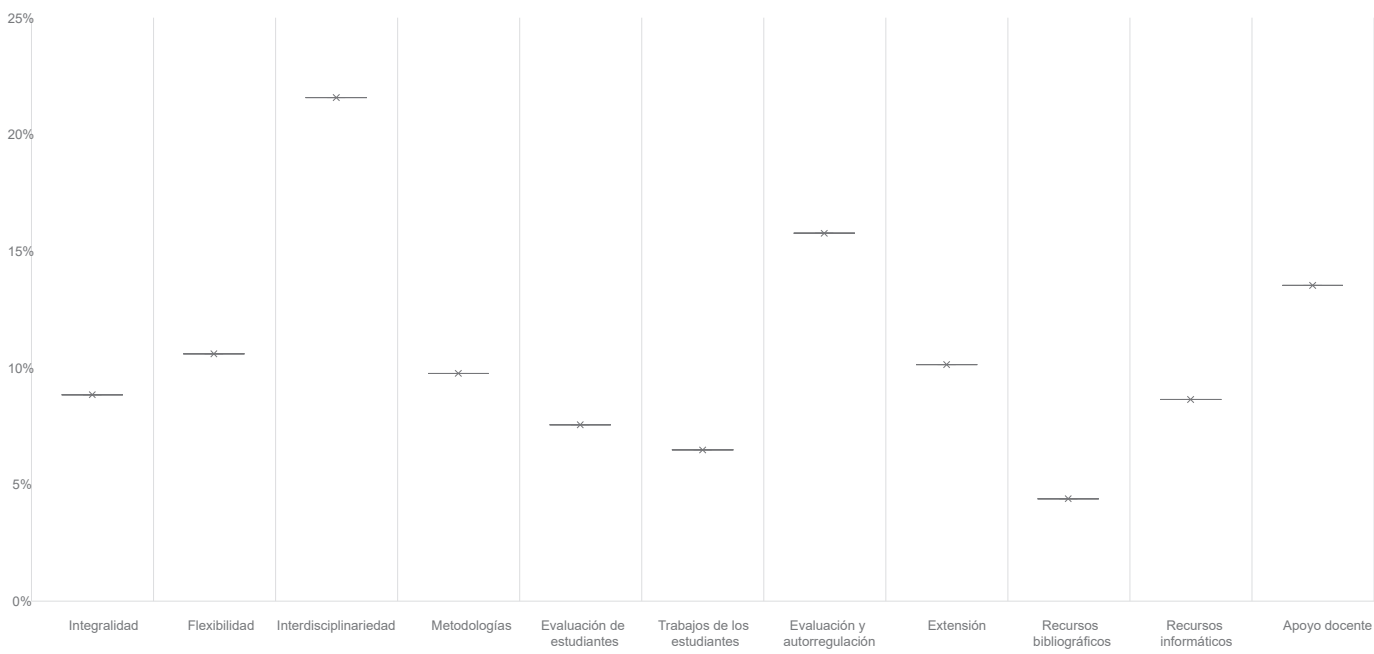

Fuente: elaboración propia.

Los pares evaluaron la interdisciplinariedad con una calificación promedio de 3.9. En algunos programas académicos la evaluación mínima fue de 2.3, y para otros programas la evaluación máxima fue de 4.7. En la característica de evaluación y autorregulación, los pares dieron una evaluación promedio de 3.92, con una calificación mínima de 3.4 y una máxima de 4.8 .

La calificación promedio de recursos bibliográficos fue de 4.4, con unas calificaciones mínimas y máximas de 4.1 y 4.6, respectivamente. Esto significó una menor dispersión de las calificaciones con respecto al promedio.

Relación entre la percepción de los estudiantes y los resultados de la evaluación externa del CNA

Las calificaciones cuantitativas tanto de estudiantes como de pares académicos presentaron diferencias en algunas de las características evaluadas; en promedio, el comportamiento de estas calificaciones fue más alto en los pares académicos, como se puede 
observar en la figura 9. En esta observamos que la línea continua que representa a los pares académicos mantuvo calificaciones en la zona de alto grado (verde claro), mientras que los estudiantes representados por la línea punteada tuvieron calificaciones tanto en la zona de alto grado como en la zona de aceptable (amarillo claro).

Figura 9. Comportamiento de las calificaciones cuantitativas entre estudiantes y pares académicos respecto al resultado global.

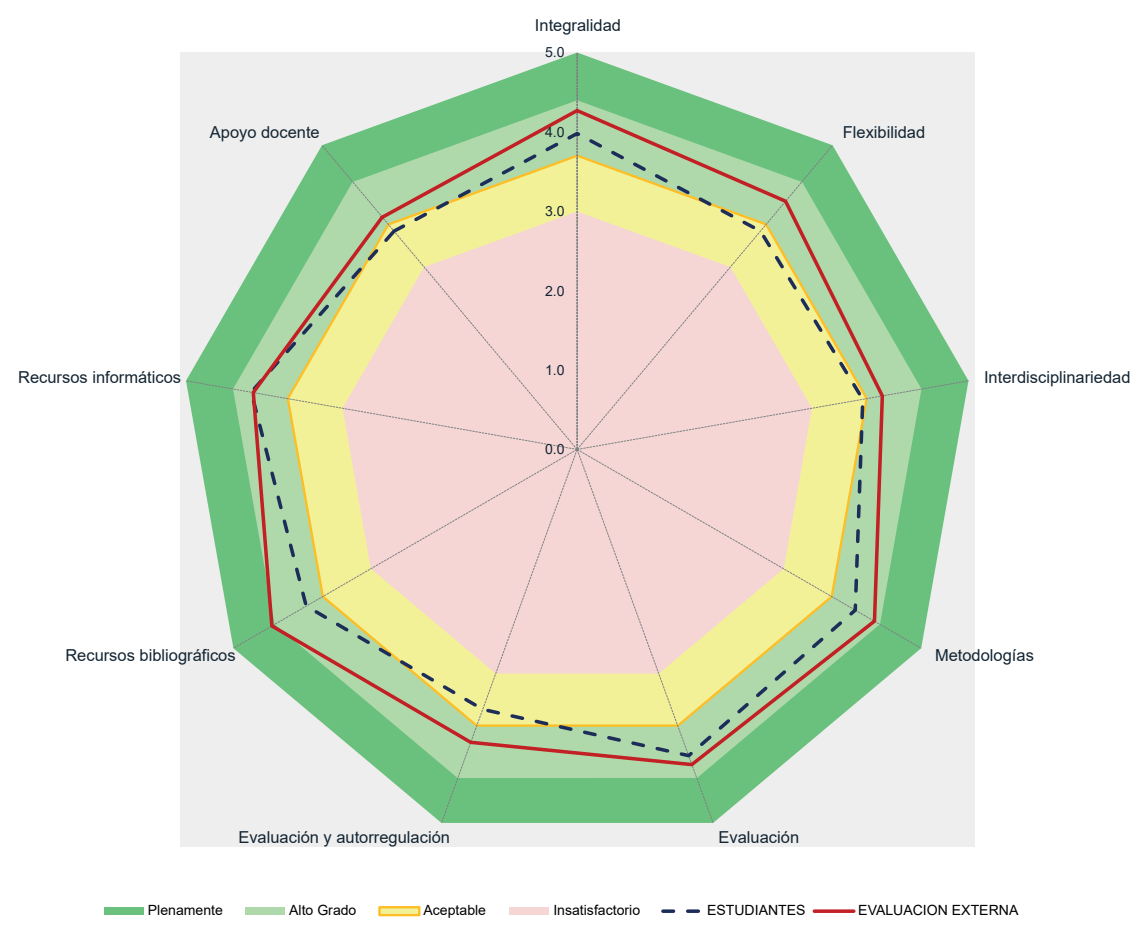

Fuente: elaboración propia.

Teniendo en cuenta los resultados no numéricos sobre la percepción de estudiantes y pares académicos en el factor de estudio, se compararon los juicios obtenidos por parte de estos estamentos con respecto al resultado global. Aquí se evaluó la concordancia en los 22 programas académicos, dado que 3 de los 25 no presentaban información suficiente en relación estos juicios. A partir de las comparaciones, se evidenció una mejor calificación en la evaluación externa con respecto a la calificación de los estudiantes. 
El $73 \%$ de los programas evaluados por los pares tuvo una calificación de alto grado, mientras que de los programas valorados por los estudiantes el $41 \%$ tuvo una evaluación insatisfactoria y un $55 \%$ una evaluación aceptable.

El índice de kappa estimado entre la percepción de los estudiantes de pregrado y la evaluación externa del CNA evidenció una concordancia débil entre las dos evaluaciones con respecto a la calidad de los procesos académicos ( $K=0.242$, aproximadamente). Por ejemplo, en los estudiantes que dieron una valoración aceptable a 12 programas, la evaluación externa presentó solo un acuerdo en un solo programa, los restantes fueron valorados como plenamente $(n=5)$ y alto grado $(n=6)$ (tabla 3$)$.

Tabla 3. Concordancia entre la percepción de los estudiantes y la evaluación externa.

\begin{tabular}{|c|c|c|c|c|c|}
\hline \multirow{2}{*}{ Estudiantes } & \multicolumn{4}{|c|}{ Evaluación externa } & \multirow{2}{*}{ Tota } \\
\hline & Plenamente & Alto grado & Aceptable & Insatisfactorio & \\
\hline Plenamente & - & - & - & - & - \\
\hline Alto grado & - & 1 & - & - & 1 \\
\hline Aceptable & 5 & 6 & 1 & - & 12 \\
\hline Insatisfactorio & - & 9 & - & - & 9 \\
\hline Total & 5 & 16 & 1 & 0 & 22 \\
\hline
\end{tabular}

Fuente: elaboración propia.

\section{Discusión}

La característica apoyo docente evidenció las mayores diferencias. La opinión de los estudiantes fue aceptable en comparación con la calificación de alto grado de los pares. Estos destacaron la consciencia institucional para el mejoramiento de los recursos de apoyo docente y los esfuerzos realizados por los programas académicos en el mantenimiento de espacios y recursos audiovisuales, tal como lo reportó Alzate-Medina (2008), quien encontró asociación entre la acreditación y el aumento de los recursos de apoyo a la docencia. 
Por otro lado, es posible que la respuesta de los estudiantes esté asociada con la percepción de una baja calidad y disponibilidad de estos recursos durante su proceso de formación, desconociendo las actividades realizadas por el programa para su mejoramiento. Este esfuerzo podría ser reconocido por los estudiantes si se utilizaran estrategias de comunicación efectivas para su conocimiento y divulgación. En su estudio, González, Berdugo y Mortigo (2017) hacen mención de que la legitimidad y la cantidad de recursos tecnológicos no garantiza que las actividades docentes y formativas sean de calidad. El uso efectivo de estos recursos depende, en buena medida, del diseño pedagógico que se prevé para ellos. Ello también es soportado por el estudio realizado por Álvarez, Rodríguez y Caro (2012), en el que se menciona que la relevancia de los apoyos institucionales para los estudiantes queda reflejada en la importancia que atribuyen a los campus virtuales, porque les proporcionan información, comunicación y aprendizaje en múltiples ámbitos.

La característica interdisciplinariedad fue calificada como aceptable por los estudiantes y de alto grado por los pares. Estas diferencias pueden deberse a que los estudiantes consideran que las actividades curriculares y extracurriculares no son pertinentes o eficaces en el enriquecimiento del programa académico, o por desconocimiento del concepto. Desde su punto de vista, la interdisciplinariedad no integra las actividades curriculares y extracurriculares que realiza la institución o el programa académico. En el estudio realizado por De León y Pinto (2017) se menciona que los estudiantes perciben que la acreditación ha impactado de forma significativa en la promoción de cursos extracurriculares, en la participación en programas sociales de ayuda a la comunidad y en distintos grupos deportivos, culturales y artísticos, pero poco ha fomentado la participación en la divulgación de conocimiento, realización de tesis y estancias de investigación.

Por su parte, los pares lo valoran de acuerdo con lo mencionado por los programas en sus informes de autoevaluación: existencia de espacios y actividades curriculares y extracurriculares con carácter explícitamente interdisciplinario, y de mecanismos que permiten 
el tratamiento de problemas pertinentes a la disciplina y al ejercicio laboral. Esto a través de orientaciones interdisciplinarias, así como de la apreciación de profesores y estudiantes sobre la pertinencia y la eficacia de la interdisciplinariedad del programa en el enriquecimiento de la calidad de este.

Este hallazgo es similar al de Alemán Rodríguez y Yera Carbonell (2011), quienes mencionan que para asumir y diseñar las acciones interdisciplinarias es necesaria la preparación de cada profesor, quien debe asumir su práctica como un proceso de investigación que domine el sistema disciplinario y las particularidades de la carrera. También destacan el trabajo cooperativo entre equipos conformados por profesores de las distintas disciplinas, que lo asuman como una forma de novedad imprescindible, y que lo evalúen continuamente para perfeccionar el trabajo. Esto favorecerá el desarrollo de la didáctica disciplinaria e interdisciplinaria.

La característica evaluación y autorregulación del programa fue calificada como aceptable por los estudiantes, probablemente porque ellos no evidencian actividades de autoevaluación que permitan mejorar aspectos relevantes del programa académico. Esto demanda una mayor participación de los estudiantes en estos procesos. Aunque se conoce el proceso que la universidad ha definido para lograr la acreditación en alta calidad, no se asocian las actividades que realizan los programas académicos en los comités de programa, consejos de facultad o instituto, comités de currículo u otros espacios donde hay participación de estudiantes, como procesos de mejoramiento continuo que llevan a la actualización de normas, reglamentaciones o cambios curriculares.

Esta característica fue calificada por los pares académicos en alto grado, debido a su conocimiento sobre los Sistemas Internos de Aseguramiento de Calidad (SIAC), que implican cambios al interior de los programas académicos, la participación de la comunidad universitaria y las mejoras continuas para ofrecer educación de alta calidad. Esto se sustenta en el estudio realizado por Alzate-Medina (2008), quien manifiesta que los estudios que se realizan sobre los resultados de la acreditación (de programas o institucional) 
en las universidades, permiten mostrar que existe un impacto positivo en la educación. Lo anterior siempre y cuando estén orientados por procesos de autoevaluación construidos participativamente y que demuestren la realidad de la institución, de manera que generen elementos para mejorar la calidad académica, los cuales son de creciente interés en la educación superior.

La característica recursos informáticos fue calificada en alto grado por parte de los estudiantes y los pares académicos. Los estudiantes otorgan valor a los sistemas, conectividad y equipos informáticos con que cuenta la universidad para el desarrollo de los procesos académicos y administrativos. Por su parte, los pares académicos reconocen los servicios, dotación de equipos y los esfuerzos de la universidad para mejorar la conectividad, así como el trabajo permanente para actualizar los recursos tecnológicos. Los estudiantes perciben que la acreditación ha impactado las condiciones tanto de infraestructura física y tecnológica del aula, propiciando así el proceso de aprendizaje, tal como lo señala De León y Pinto (2017).

En relación con la característica recursos bibliográficos, tanto los estudiantes como los pares otorgaron la calificación de alto grado. Esta evaluación se asocia con la disponibilidad de los recursos bibliográficos, de las nuevas tecnologías y el rápido acceso a la información. En su estudio, García (2017) menciona que las oportunidades de mejora más frecuentes tanto de programas acreditados como reacreditados corresponden a la necesidad de mejorar los recursos bibliográficos. Por esta razón hay esfuerzos institucionales importantes para la adquisición de material bibliográfico, que se evidencia en la valoración otorgada por los estudiantes y los pares académicos.

Por lo tanto, se pretende analizar la coherencia de los procesos de autoevaluación como práctica permanente, la participación de la comunidad académica, la percepción que sobre las instituciones tienen los evaluadores externos, así como la ejecución de los planes de mejoramiento. Todo lo anterior como garantía del aseguramiento de la calidad. 


\section{Limitaciones}

En la visita de evaluación externa realizada por los pares académicos se observó que, a pesar de contar con los lineamientos del CNA y conocer los procesos a seguir para la evaluación de un programa académico, no existe homogeneidad al momento de presentar el informe. No todos los pares emitieron calificación por cada una de las características, solo en seis programas se emitieron calificaciones para cada una, lo que dificultó el análisis comparativo y detallado de la verificación de los resultados expresados en el informe de la evaluación externa del programa.

Esta limitación ha sido reportada por otros estudios que registran que una de las falencias del sistema de acreditación colombiano reside en la preparación de los pares académicos. Por esta razón hay debilidades en la ponderación y evaluación de la información contenida en una autoevaluación (Gómez, 2016).

\section{Conclusiones}

Este estudio evidencia que existe una baja concordancia entre la percepción de los estudiantes y los pares académicos frente al factor procesos académicos. Los estudiantes responden a la autoevaluación a partir de su experiencia a lo largo de su proceso formativo que, en algunos casos, puede estar sesgada por la desinformación, la falta de claridad o de conocimiento. Los pares, por su parte, tienen una visión integral del proceso de autoevaluación, que hace énfasis en los esfuerzos y actividades desarrolladas por la institución para atender las oportunidades de mejora identificadas en los planes de mejoramiento. Este aspecto es identificado con gran detalle y precisión por los pares, pero no necesariamente es evidente para los estudiantes. En todos los casos, la valoración fue más alta para los pares que para los estudiantes.

Para llevar a cabo procesos de mejoramiento continuo que permitan a los estudiantes modificar su percepción en relación con la calidad 
de los procesos académicos, se hace necesario analizar los resultados obtenidos y realizar estrategias que permitan identificar las acciones realizadas por la institución y los programas para que sean conocidas por los estudiantes. Este aspecto sí es valorado por los pares académicos.

En la evaluación de las instituciones existen varios aspectos con los cuales se analiza el grado de calidad; sin embargo, son los procesos académicos los que adquieren mayor relevancia. Dichos procesos identifican a la comunidad académica con la misión, visión, identidad y objetivos institucionales, y la manera como estos orientan la formación integral, flexible, actualizada e interdisciplinar de los futuros profesionales que buscan las organizaciones.

Se hace necesario realizar otros estudios, en los cuales se tenga en cuenta la opinión de los docentes sobre estas características. Esto podría confirmar la necesidad de realizar actividades para la socialización de los conceptos relacionados con la política curricular y los procesos de autoevaluación. Se considera importante profundizar también en la comprensión del impacto que el factor tiene en los programas académicos y en el sistema de educación superior nacional.

\section{Recomendaciones}

A nivel institucional, se plantea la importancia de realizar una socialización con los estudiantes sobre los conceptos que incorporan los procesos académicos: integralidad, interdisciplinariedad y flexibilidad, siendo coherentes con los lineamientos del proyecto institucional. Con ello, se busca que los estudiantes logren una mayor claridad y conocimiento de los aspectos que constituyen su formación integral, las estrategias utilizadas por la universidad para llevarlos a cabo y las actividades en que participan los estudiantes sin ser conscientes de su correspondencia con la integralidad e interdisciplinariedad. Este aspecto está articulado con el factor de misión y visión institucionales. 
También se requiere fortalecer en la comunidad universitaria el conocimiento del Sistema Interno de Aseguramiento de la Calidad y las actividades relacionadas con los procesos de autoevaluación, autorregulación y mejoramiento continuo. Esto con el propósito de ampliar la visión de que las actividades que se realizan al interior de los programas, unidades académicas, facultades, institutos y a nivel institucional —con el compromiso y participación de todos los involucrados (estudiantes, docentes, directivos, egresados, personal administrativo y empleadores) - , hacen parte de las mejoras para alcanzar la calidad educativa que merece la sociedad. De esta manera se busca identificar que, más allá del proceso técnico, es importante la apropiación de lo que implica la autoevaluación para la institución.

\section{Referencias}

Aguerrondo, I. (1993). La calidad de la educación: ejes para su definición y evaluación. Revista interamericana de desarrollo educativo, 37(116), 561-578. http://formaciondocente.com.mx/Bibliotecadigital/18_ TemasEducacion/La\%20Calidad\%20de\%20la\%20Educacion.pdf

Alemán Rodríguez, R. y Yera Carbonell, G. D. C. (2011). La interdisciplinariedad en ciencias médicas y la matemática. Revista Cubana de Higiene y Epidemiología, 49(3), 490-498. http://scielo.sld.cu/ scielo.php?script $=$ sci_arttext\&pid=S1561-30032011000300016

Álvarez Arregui, E., Rodríguez Martín, A. e Inda Caro, M. (2012). Percepciones de los estudiantes universitarios sobre elección de la carrera, los apoyos institucionales y la docencia en la licenciatura de Pedagogía. Aula abierta. http://digibuo.uniovi.es/dspace/bitstream/ 10651/10893/4/Aula\%20abierta.pdf

Alzate-Medina, G. M. (2008). Efectos de la acreditación en el mejoramiento de la calidad de los programas de psicología de Colombia. Universitas Psychologica, 7(2),425-439. https://www.redalyc.org/ pdf/647/64770210.pdf 
Bonfante, L. A. P. (2019). Análisis de la calidad de la educación superior de Colombia. Criterio Libre, 18(31), 187-205. https://dialnet.unirioja.es/ servlet/articulo?codigo $=7340735$

Calvo, J. F. (2018). Calidad educativa en la educación superior colombiana: una aproximación teórica. Sophia, 14(2), 4-14. https://dialnet.unirioja. es/servlet/articulo?codigo $=6522053$

Colmenares Delgado, O. A. y Saavedra Torres, J. L. (2007). Aproximación teórica de los modelos conceptuales de la calidad del servicio. Técnica administrativa, 6(32), 2. http://www.cyta.com.ar/ta0604/v6n4a2.htm

Consejo Nacional de Acreditación (CNA). (2013). Lineamientos para la acreditación de programas de pregrado. https://www.cna.gov.co/1741/ articles-186359_pregrado_2013.pdf

Cuevas López, M., Fernández Cruz, M., Díaz Rosas, F., Gijón Puerta, J., Lizarte Simón, E.J., IbáñezCubillas, P., El Homrani, M., Ávalos Ruiz, I. y Rodríguez Muñoz, R. (2019). Liderazgo y calidad en la Educación Superior. Edmetic, Revista de Educación Mediática y TIC, 8(2), 52-72. https://doi. org/10.21071/edmetic.v8i2.12120.

De León, E. M. y Pinto, Z. R. (2017). El impacto de la acreditación en la mejora de la calidad de los programas educativos que ofrece la universidad autónoma de Tamaulipas: un estudio de caso. Revista Iberoamericana de Evaluación Educativa, 10(2), 65-83.

Estrada, L. R. (1999). Hacia un modelo de evaluación de la calidad de instituciones de educación superior. Revista iberoamericana de educación, (21), 93-103. https://rieoei.org/historico/documentos/rie21 a06.PDF

Ferrer, A. T. (2006). La evaluación de la calidad de la educación: conceptos, modelos e instrumentos. Transatlántica de educación, (1), 19-30. https://dialnet.unirioja.es/servlet/articulo?codigo $=2346178$ 
Gallego, J. D. (2012). Línea de investigación: currículo y evaluación educativa. Revista Interamericana de Investigación, Educación y Pedagogía (RIIEP), 5(2). https://revistas.usantotomas.edu.co/index. php/riiep/article/view/1323/1521

García, J. M. (2017). Resultados de acreditación obtenidos mediante seguimiento: una aproximación al estudio del impacto de la acreditación. Congreso Universidad, 5(1), 16. https://www.sinaes.ac.cr/documentos/ Productos_de_Investigacion_Academica/Publicacion_en_revista/ Resultados_de_la_acreditacion_obtenidos_mediante_seguimiento.pdf

Giraldo, U., Abad, D. y Díaz, E. (2007). Bases para una política de calidad de la educación superior en Colombia. https://www. cna. gov. co/1741/ articles-186502_doc_academico10. Pdf

Gómez, V. (2016). Crisis de sentido y falencias metodológicas del sistema de acreditación colombiano. http://www.universidad.edu.co/images/ cmlopera/descargables/crisisacreditacion.pdf

González Guerrero, K., Berdugo Silva, N. C. y Mortigo Rubio, A. (2017). Incidencia de los entornos virtuales de aprendizaje en la calidad de la educación superior, desde el contexto colombiano. Revista Interamericana de Investigación, Educación y Pedagogía (RIIEP), 10(2), 11-24. https://doi.org/10.15332/s1657-107X.2017.0002.01

Ramírez, E. A. (2011). La investigación cualitativa en educación. Balance y retos en el contexto colombiano. Revista Interamericana de Investigación, Educación y Pedagogía (RIIEP), 4(1), 81-91. https://doi. org/10.15332/s1657-107X.2011.0001.05

Ramos, C. B. (2007). Un análisis crítico sobre los modelos de gestión de la calidad en la educación. Innovación educativa, 7(41), 19-29. https:// www.redalyc.org/pdf/1794/179421215003.pdf

Real Academia Española. (2014). Diccionario de la lengua española

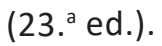


Reyes Sánchez, O. y Reyes Pazos, M. (2012). Percepción de la Calidad del Servicio de la Educación Universitaria de Alumnos y Profesores (Higher Education Quality-A Student and Profession Perception). Revista internacional administración \& finanzas, 5(5), 87-98. https:// papers.ssrn.com/sol3/papers.cfm?abstract_id=2095414

Ruiz, A. E., de los Ángeles Calderón, M., Figueroa, H. M., Guerrero, F. A. y Bastidas, J. A. (2015). Hacia un mejoramiento de los procesos académicos delCentro deldiomas de la Universidad de Nariño, Colombia. Revista Hechos y Proyecciones del Lenguaje, (21). 62-77. https://www.researchgate. net/profile/Jesus_Bastidas2/publication/326522368_Hacia_un_mejor amiento_de_los_procesos_academicos_del_Centro_de_Idiomas_de_ la/links/5b525e160f7e9b240ff279eb/Hacia-un-mejoramiento-de-losprocesos-academicos-del-Centro-de-Idiomas-de-la.pdf

Saumeth, K. M., Afanador, T. S., Ospino, L. S. y Barraza, F. M. (2012). Calidad y su evolución: una revisión. Dimensión empresarial, 10(2), 100107. https://dialnet.unirioja.es/servlet/articulo?codigo $=4400435$

Selley, O. D. y Ramírez, R. E. (2018). Evaluación y acreditación desde la perspectiva de las Universidades: el Consejo de Evaluación y Acreditación Internacional. Universidades, 69(78), 65-74. https://www.redalyc.org/ pdf/3606/360657469023.pdf

Tünnermann, C. (2006). Pertinencia y calidad de la educación superior. http://iep.udea.edu.co:8180/jspui/bitstream/123456789/258/1/ Pertinencia\%20Calidad\%20Educacion\%20Superior\%20-CarlosTunnermann. pdf 